EDITORIAL

\title{
Cohort studies investigating the effects of exposures: key principles that impact the credibility of the results
}

(c) The Author(s), under exclusive licence to The Royal College of Ophthalmologists 2021

Eye (2022) 36:905-906; https://doi.org/10.1038/s41433-021-01897-0

\section{WHAT ARE COHORT STUDIES?}

Cohort studies are observational studies that follow groups of patients with different exposures forward in time and determine outcomes of interest in each exposure group or that investigate the effect of one or more participant characteristics on prognostic outcomes [1]. The focus of this editorial is on cohort studies that investigate the effects of exposures that may be associated with an increased or a decreased occurrence of the outcome of interest. Cohort studies may be prospective or retrospective in design. In prospective cohort studies, investigators enroll participants, assess exposure status, initiate follow up, and measure the outcome of interest in the future. In retrospective cohort studies, data on both the exposures and outcome of interest have been previously collected.

\section{PURPOSE OF COHORT STUDIES}

While large well-designed randomized controlled trials (RCTs) represent the optimal design for making inferences about the effects of exposures or interventions on health outcomes, they are often not feasible to conduct-due to costs or challenges of recruiting patients with rare conditions and following patients for sufficient durations. Further, patients included in RCTs may not be representative of patients encountered in practice and the effectiveness of therapies in strict clinical trials may be different than when implemented in routine practice. In such circumstances, well-designed observational studies, which include cohort studies, can play an important role in producing evidence to guide clinical care decisions in ophthalmology. Cohort studies can also be conducted to generate hypotheses and establishing questions for future RCTs.

The differentiating characteristics between observational (e.g., cohort study) and experimental (e.g., RCT) study designs are that in the former the investigator does not intervene and rather "observes" and examines the relationship or association between an exposure and outcome. Examples of cohort studies in ophthalmology include evaluation of a possible association between exposure to ambient air pollution and age-related cataract [2]; or assessment of the impact of eye preserving therapies for patients with advanced retinoblastoma [3].

\section{KEY DETERMINANTS OF CREDIBILITY (I.E., INTERNAL VALIDITY) IN COHORT STUDIES}

Readers considering applying evidence from cohort studies should be mindful of the following factors that affect the credibility or internal validity of cohort studies.

\section{Factors that decrease the credibility of cohort studies}

Cohort studies are at serious risk of confounding bias and so adjusting or accounting for confounding factors is a priority in these studies. Confounding occurs when the exposure of interest is associated with another factor that also influences the outcome of interest. Investigators can use various design (e.g., matching) and statistical methods (e.g., adjusted analyses based on regression methods) to deal with known, measured confounders. Readers should assess whether the authors accounted for known confounders of the relationship under investigation in either their design or statistical analysis. Readers should be mindful, however, that possibility of residual confounding caused by unknown or unmeasured confounders always remains.

Inappropriate selection of participants into the cohort study can result in selection bias. Selection bias occurs when selection of participants is related to both the intervention and outcome. Bias in measurement of exposure/outcome, or detection bias, can arise when outcome assessors are aware of intervention status, different methods are used to assess outcomes in the different intervention groups, and/or the exposure status is misclassified differentially or non-differentially (i.e., the probability of individuals being misclassified is different or equal between groups in a study, respectively).

Missing data may also affect the credibility of cohort studies. Bias due to missing data in prospective and retrospective studies arises when follow up data are missing for individuals initially included in the study. Participants with missing outcome data may differ importantly from those with complete data (e.g., they may be healthier or may not have experienced adverse events).

Last, credibility of a cohort study may be affected by the reporting of results. Selective reporting arises when investigators selectively report results in studies in such a way so that the study report highlights or emphasizes evidence supporting a particular hypothesis and does not report or understates evidence supporting an alternative hypothesis. Investigators may selectively report results for timepoints or measures that produced results consistent with their preconceived beliefs or results that were newsworthy and disregard results for timepoints or measures that produced results that were inconsistent with their beliefs or considered not newsworthy. Publication bias refers to the propensity for studies with anomalous, interesting, or statistically significant results to be published at higher rates or to be published more rapidly or to be published in journals with higher visibility.

\section{Factors that increase the credibility of cohort studies}

Three uncommon situations can sometimes make us more certain of findings of cohort studies-in some circumstances, these situations can make us as confident of evidence from cohort studies as we would be for evidence from a rigorous RCT. First, when the observed effect is large (typically a relative risk $(\mathrm{RR})>2$ or $\mathrm{RR}<0.5$ ), biases, such as confounding, are less likely to completely explain the observed effect. Second, we may be more certain of results when we observe a dose-response gradient: biases in non-randomized studies (e.g., confounding and errors in the classification of the exposure) are unlikely to produce spurious dose-response associations., when all suspected biases are believed to act against the 
observed direction of effect, we can be more certain that the observed effect is not due to the suspected biases. It is, however, difficult to anticipate with sufficient certainty the direction in which effects are likely biased in complex epidemiological studies. Because situations that make us more certain of findings of cohort studies occur infrequently, cohort studies usually provide only low to very low certainty evidence [4].

\section{APPLICABILITY (I.E., EXTERNAL VALIDITY) IN COHORT STUDIES} If the populations, exposures, or outcomes investigated in cohort studies differ from the those of interest in routine or typical settings, the evidence may not be applicable or externally valid. Such judgements depend on whether differences between studies and the question of interest would lead to an appreciable change in the direction or magnitude of effect. Generally, observational studies (e.g., cohort studies) have higher external validity than experimental studies (e.g., RCTs) [5].

\section{CONCLUSION}

Cohort studies follow a population exposed or not exposed to a potential causal agent forward in time and assess outcomes. Cohort studies are beneficial because these studies allow the investigators to observe a possible association between an exposure and outcome of interest in a population that cannot be randomly subjected to an exposure due to ethical, methodological, or feasibility limitations. Cohort studies, however, have several limitations that should be acknowledged and minimized if possible.

Anna Miroshnychenko ${ }^{1}$, Dena Zeraatkar ${ }^{1,2}$, Mark R. Phillips ${ }^{1}$ Sophie J. Bakri ${ }^{3}$, Lehana Thabane (iD ${ }^{1,4}$, Mohit Bhandari (iD ${ }^{1,5}$, Varun Chaudhary (D ${ }^{1,5 凶}$ and for the Retina Evidence Trials InterNational Alliance (R.E.T.I.N.A.) Study Group*

${ }^{1}$ Department of Health Research Methods, Evidence, and Impact, McMaster University, Hamilton, ON, Canada. ${ }^{2}$ Department of Biomedical Informatics, Harvard Medical School, Boston, MA, USA. ${ }^{3}$ Department of Ophthalmology, Mayo Clinic, Rochester, MN, USA. ${ }^{4}$ Biostatistics Unit, St. Joseph's Healthcare-Hamilton, Hamilton, ON, Canada. ${ }^{5}$ Department of Surgery, McMaster University, Hamilton, ON, Canada. ${ }^{*} A$ list of authors and their affiliations appears at the end of the paper. ${ }^{\bowtie}$ email: vchaudh@mcmaster.ca

\section{REFERENCES}

1. Barrett D, Noble H. What are cohort studies? Evid-Based Nurs. 2019;22:95-6.

2. Shin J, Lee $\mathrm{H}, \mathrm{Kim} \mathrm{H}$. Association between exposure to ambient air pollution and age-related cataract: a nationwide population-based retrospective cohort study. Int J Environ Res Public Health. 2020;17:9231.

3. Zhou C, Wen X, Ding Y, Ding J, Jin M, Liu Z, et al. Eye-preserving therapies for advanced retinoblastoma: a multicenter cohort of 1678 patients in China. Ophthalmology.2021;50161-6420:00683-7.

4. Guyatt GH, Oxman AD, Vist G, Kunz R, Brozek J, Alonso-Coello P, et al. GRADE guidelines: 4. Rating the quality of evidence-study limitations (risk of bias). J Clin Epidemiol. 2011;64:407-15.

5. Rothwell PM. External validity of randomised controlled trials: "to whom do the results of this trial apply?". Lancet.2005;365:82-93.

\section{AUTHOR CONTRIBUTIONS}

AM was responsible for writing, critical review and feedback on manuscript. DZ was responsible for writing, critical review and feedback on manuscript. MRP was responsible for conception of idea, critical review and feedback on manuscript. SJB was responsible for critical review and feedback on manuscript. LT was responsible for critical review and feedback on manuscript. MB was responsible for conception of idea, critical review and feedback on manuscript. VC was responsible for conception of idea, critical review and feedback on manuscript.

\section{COMPETING INTERESTS}

SJB: Consultant: Adverum, Allegro, Alimera, Allergan, Apellis, Eyepoint, ilumen, Kala, Genentech, Novartis, Regenexbio, Roche, Zeiss - unrelated to this study. MB: Research funds: Pendopharm, Bioventus, Acumed - unrelated to this study. VC: Advisory Board Member: Alcon, Roche, Bayer, Novartis; Grants: Bayer, Novartis unrelated to this study. Rest authors have nothing to disclose.

\section{ADDITIONAL INFORMATION}

Correspondence and requests for materials should be addressed to Varun Chaudhary.

Reprints and permission information is available at http://www.nature.com/ reprints

Publisher's note Springer Nature remains neutral with regard to jurisdictional claims in published maps and institutional affiliations.

\section{FOR THE RETINA EVIDENCE TRIALS INTERNATIONAL ALLIANCE (R.E.T.I.N.A.) STUDY GROUP}

Varun Chaudhary $\mathbb{D}^{5}$, Mohit Bhandari $\mathbb{D}^{5}$, Charles C. Wykoff ${ }^{6,7}$, Sobha Sivaprasad ${ }^{8}$, Lehana Thabane $\mathbb{D}^{1,4}$, Peter Kaiser ${ }^{9}$, David Sarraf ${ }^{10}$, Sophie J. Bakri, Sunir J. Garg ${ }^{11}$, Rishi P. Singh ${ }^{12,13}$, Frank G. Holz ${ }^{14}$, Tien Y. Wong ${ }^{15,16}$ and Robyn H. Guymer ${ }^{17,18}$

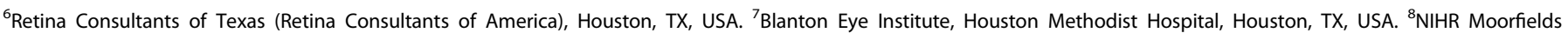

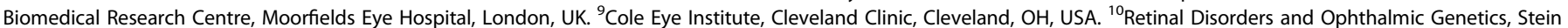

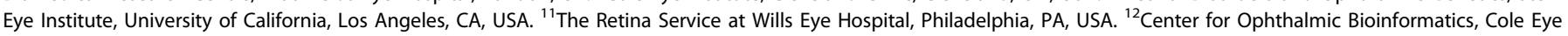
Institute, Cleveland Clinic, Cleveland, OH, USA. ${ }^{13}$ Cleveland Clinic Lerner College of Medicine, Cleveland, OH, USA. ${ }^{14}$ Department of Ophthalmology, University of Bonn, Boon,

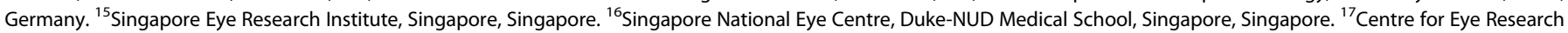

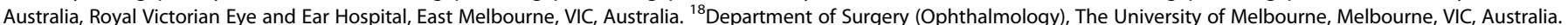

